possibility that the plant has been introduced lately cannot be excluded. However, the species grows in natural vegetation on limestone rocks. Plants in the vicinity include Omphalodes commutata, Rubia peregrina, Linaria platycalyx, Scilla peruviana, Jasminum fruticans, Fumaria capreolata and Euphorbia pinea. The altitude is ca. $810 \mathrm{~m}$. The place was visited also during an excursion in May 1984, and the species was then still present.

The white-flowered M. speciosa is immediately distinguished from other European species with white flowers by its incised-dentate stipules and 1arger, 7-8 mm long corolla. The species is known previously only from Morocco and northern Algeria.

\title{
COMENTARIOS SOBRE ALGUNAS PLANTAS NUEVAS PARA LA FLORA DE AVILA, ESPAÑA
}

\author{
María Andrea CARRASCO \& Javier ESTRADA
}

RESUMEN: Se presentan siete taxones nuevos para la flora de Avila, incluyendo algunos comentarios sobre su distribución en el centro peninsular.

SUMMARY: In this paper, seven taxa recorded for the first time from Avila province are listed. We present also, some commentaries about its distribution in the centre-west of Spain.

Con objeto de aportar datos para el mejor conocimiento de la flora vascular del centro de la peninsula, hemos realizado herborizaciones al suroeste del Sistema Central, en la cuenca alta del rio Corneja, tributarió del Tormes, perteneciente a la Cuenca del Duero.

E1 territorio se sitúa en el sector Cornejano-Amblense de la provincia corológica Carpetano-Ibérico-Leonesa, a altitudes que oscilan entre 1000 y 1600 m.s.m., con una oscilación térmica anual superior a $20^{\circ} \mathrm{C}$, lo que nos indica la 
continentalidad del clima.

Geológicamente se sitúa en el tramo Galaico-Castellano del Macizo Herpérico, ocupando la fosa tectónica del Valle de Corneja. Los procesos de metamorfismo de edad hercínica en estos sustratos, formados básicamente por granitos porfídicos y anatexitas, han dado lugar a suelos ácidos que se incluyen en las tierras pardas húmedas sobre granitos. En Villafranca de la Sierra, donde se recogen los taxones citados, contactan las arcillas rojas y margas del neógeno con los granitos adamelitivos de dos micas.

Todos lo's taxones citados representan al menos primeras citas provinciales. Sus testimonios están depositados en el Herbario MACB (Herbario de la Facultad de Biología de la Universidad Complutense). A este herbario nos veniamos refiriendo en anteriores trabajos como MAC, siglas que corresponden a uno del Brasil.

Equisetum hyemale L.

Avila: Villafranca de la Sierra, arroyo de Pinonegrillo, 30T UK1284, 18-VII-1984, Carrasco \& Estrada, MACB 20324.

En su reciente estudio sobre el género, Prada (1986:25) no incluye localidad abulense, aunque sí lo señala de otras muchas provincias al norte de la Península.

Nuestros materiales han sido amablemente revisados por C. Prada, monógrafa del género.

Añádase Av a la corología que presenta Flora Ibérica, I.

Fumaria officinalis L. subsp. officinalis

Avila: Villafranca de la Sierra, bajo matorrales entre encinas, 30T UK1185, 16-V-1986, Carrasco \& Estrada, MACB 20323.

Lidén (1986:463) en su revịsión del género, no recoge la provincia de Avila entre las que señala para las distintas subespecies de F. officinalis L. F. officinalis L. subsp. officinalis es nueva para Avila y también la conocemos de Cáceres, provincia de la que la reparten Ladero, Santos \& González, que la recogen en Almaraz (MACB 12794).

Añadanse Av y Cc a la corología que presenta Flora Ibérica, I.

Trifolium hirtum All.

Avila: Villafranca de la Sierra, pastizales de terófitos sobre suelos arenosos, 30T UK1186, 24-V-1985, Carrasco \& Estrada, MACB 20322.

En el centro peninsular es especie conocida de antiguo, como 10 atestiguan las citas de Madrid, Valladolid y Guadalajara que recoge Vicioso (1951:323) y Salamanca (Lainz, 1955).

Recientemente existen testimonios que amplian su área de distribución a Segovia, de donde la señala Romero Martín (1987:261). Creemos que no había sido citada para Avila.

Bupleurum gerardi All.

Avila: Villafranca de la Sierra, en claros de encinar sobre suelos pedregosos, a $1250 \mathrm{~m} \mathrm{s.m.,} \mathrm{30T} \mathrm{UK1287,} \mathrm{7-VII-1986,} \mathrm{Carrasco} \mathrm{\&} \mathrm{Estrada,} \mathrm{MACB}$ 20321.

No había sido señalado con anterioridad para Avila, aunque ya Ladero \& Velasco (1978:506), indican que B. gerardi subsp. filicaule (Brot.) P. Couth., taxon al que pertenecerían nuestros materiales, se extiende por la provincia corológica Carpetano-Ibérico-Leonesa, recogiendo citas de provincias limítrofes con la nuestra, como Madrid, Salamanca y Toledo.

Más recientemente, Cebolla \& al. (1982:229), la señalan de Segovia, en Sepúlveda. 
Clinopodium vulgare L. subsp. arundanum (Boiss.) Nyman

Avila: Villafranca de la Sierra, entre rodales de Quercus rotundifolia Lam., 30T UK1186, 4-VII-1985, Carrasco \& Estrada, MACB 20326.

No citada con anterioridad para Avila, sí se conocía de otras provincias cercanas como Cáceres (Carrasco, MACB 13215 al 13218) y Segovia (Romero Martín, Sala 35003 y 35004).

Brachypodium distachyon (L.) Beauv.

Avila: Villafranca de la Sierra, en claros de encinar sobre suelos pedregosos, a $1250 \mathrm{~m} \mathrm{s.m.,} \mathrm{30T} \mathrm{UK1287,} \mathrm{7-VII-1986,} \mathrm{Carrasco} \mathrm{\&} \mathrm{Estrada,} \mathrm{MACB}$ 20325.

Rara en el centro-oeste peninsular, de donde sólo conocemos la cita de Laínz (1955:494) que la señala por primera vez para Salamanca y la de Cebolla \& al. (1985:207) en Segovia.

Orchis morio L. subsp. picta (Loisel.) Arcangeli

Avila: Villafranca de la Sierra, sotobosque en el robledal, 30T UK1283, 28-V-1986, Cardiel, Carrasco \& Estrada, MACB20327.

En el centro-oeste español la recoge con anterioridad Fernández Díez en Alba de Tormes en la provincia de Salamanca (Casaseca \& al. 1981); Carrasco (1981:46) la cita en Plasencia en la provincia de Cáceres; y Cebolla \& al. (1985:212) la señala en Segovia: Villar de Sobrepeña.

\section{BIBLIOGRAF IA}

CARRASCO, M.A. -1981- Notas florísticas del Valle del Jerte (Cáceres), Trab. Dep. Bot. Fisiol. Veg. 11:33-47.

CASASECA, B., FERNANDEZ DIEZ, F.J., AMICH, F., RICO, E. \& SANCHEZ, J . -1981- Flora española. Segunda centuria. Universidad de Salamanca.

CEBOLla, C., RIVAS, M.A. \& SORIANO, C. -1982- Catálogo florístico del Cañón del río Duratón (Segovia, España) II. Rosaceae a Umbelliferae. Lazaroa 4:227-240.

CEBOLlA, C., RIVAS, M.A. \& SORIANo, C. -1985- Catálogo florístico del Cañón del río Duratón (Segovia, España), V. Alismataceae a Orchidaceae. Lazaroa 6:205-215.

LADERO, M. \& VELASCO, A. -1978-Adiciones a la flora de los Montes de Toledo, Anales Inst. Bot. A.J. Cavanilles 34(2):497-519.

LAINZ, M. -1955- Contribución al catálogo de la flora salmantina, Anales Inst. Bot. A.J. Cavanilles 13:469-498.

LIDEN, M. -1986- Fumaria L. in S. Castroviejo et al. (ed.). Flora Ibérica, I. Madrid.

PRADA, C. -1986- Equisetum L. in S. Castroviejo et al. (ed.). Flora Ibérica, I. Madrid.

ROMERO MARTIN, T. -1987- Flora y vegetación de la cuenca del río Duratón. Tesis Doctoral (Inéd.). Salamanca.

VICIOSO, C. -1951- Tréboles españoles. Revisión del género Trifolium, Anales Inst. Bot. A.J. Cavanilles 11(2):298-383.

(Aceptado para su publicación el 10 de septiembre de 1987)

Dirección de los autores: Departamento de Biología Vegetal, I. Facultad de Biología. Universidad Complutense, 28040, Madrid. 